\title{
REFERENCE
}

NISTIR 6638

\section{Automated Luminance-Meter Control Software for Electronic-Display Measurements}

\author{
J. Matthew Treinen
}

$Q C$ 


\title{
Automated Luminance-Meter Control Software for Electronic-Display Measurements
}

\author{
J. Matthew Treinen \\ Optoelectronics Division \\ Electronics and Electrical Engineering Laboratory \\ National Institute of Standards and Technology \\ 325 Broadway \\ Boulder, CO 80305
}

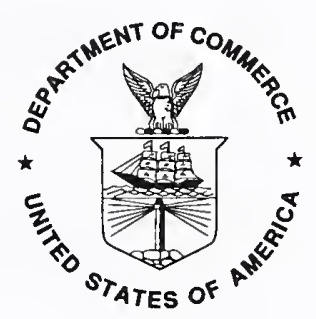

U.S. DEPARTMENT OF COMMERCE

Carlos M. Gutierrez, Secretary

TECHNOLOGY ADMINISTRATION

Michelle O'Neill, Acting Under Secretary for Technology

NATIONAL INSTITUTE OF STANDARDS AND TECHNOLOGY

William A. Jeffrey, Director 



\title{
Automated Luminance-Meter Control Software For Electronic-Display Measurements
}

\author{
J. Matthew Treinen \\ National Institute of Standards and Technology \\ Optoelectronics Division \\ 325 Broadway \\ Boulder, CO 80305
}

To be able to make automated measurements of light sources and displays, the Flat Panel Display Laboratory developed National Instrument's LabVIEW software to control a Minolta LS-100/1 10 luminance meter. The Virtual Instrument controls all functionalities of the luminance meter, while also providing the ability to take continuous measurements at set time intervals and to save the luminance data to file.

Keywords: Key words: display measurements, graphical user interface software, laboratory automation, LabVIEW, luminance measurement, luminance meter control, Minolta LS-100, Minolta LS-1 10, National Instruments, Virtual Instrument

\section{Introduction}

The Flat Panel Display Laboratory of the Display Metrology Project (NIST Sources, Detectors, and Displays Group) needs to make automated measurements of light sources and displays. Because software was not currently available to perform such measurements using the Minolta LS-100/110 luminance meters [1], we needed to create our own. The luminance meters can be controlled by a remote computer through an RS-232 serial cable connection. Using National Instrument's LabVIEW software [1], a user interface for the detector is constructed through this serial connection. In addition to providing computer controllability for all of the functionality of the detector, the LabVIEW Virtual Instrument (VI) also allows data to be saved to file and measurements to be taken continuously at fixed time intervals.

The Virtual Instrument is separated into two files: the main file, titled "LS-100 Luminance Detector Controller.vi," and a sub-VI called within the instrument and titled "Luminance Detector Update Settings;" it operates on systems with LabVIEW 6.1 or higher installed.

To help the reader understand the operation and use of the LabVIEW VI, I explain the organization and structure of the virtual instrument diagram and describe the user operation and error handling of the different features.

\section{Organization/Structure of Virtual Instrument}

The Virtual Instrument operates in a sequence of three steps. First, it configures the serial connection. Then the VI advances into the operational stage. The instrument is designed to continue running within the operational stage until the user clicks the "Stop/Abort Program" button. When this happens, the program closes the serial port and ceases operation. Each stage's operation is detailed below. 


\section{1 Configuration Stage}

The first step in the instrument operation is to configure the VISA communication session within LabVIEW. VISA is an instrument controller interface within LabVIEW. Using the settings from the LS100/110 Communications Manual, which are detailed in Table 1, and the "VISA Configure Serial Port" VI, the serial port is configured to the required communication settings for the LS-100/110. This configuration step is shown in Figure 1, which is in the " 0 " sequence step in the overall VI diagram.

Table 1. LS-100/110 Communications parameters*

\begin{tabular}{lr}
\hline Baud rate & 4800 \\
Data length & 7 bits \\
Parity & EVEN \\
Stop bit & 2 bits \\
\hline *From luminance meter communications manual [2]
\end{tabular}

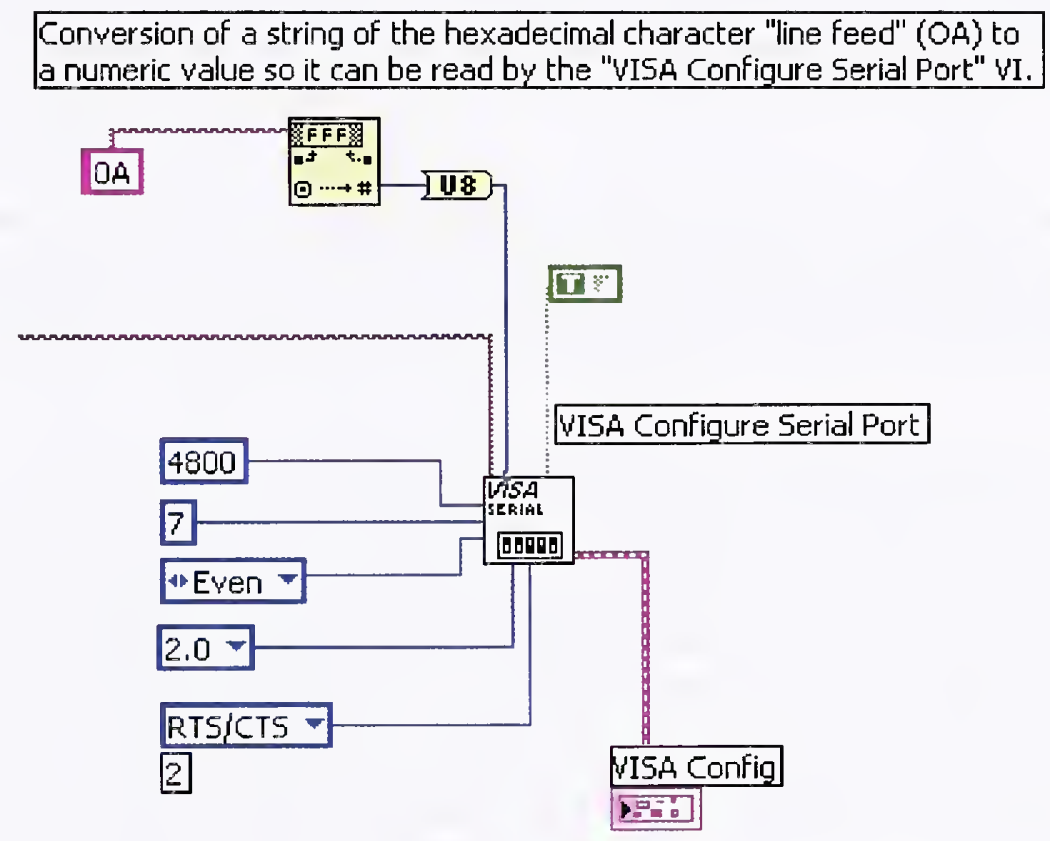

Figure 1. "VISA Configure Serial Port" VI settings.

One important difference in configuration to note between the LS-100/110 configuration and the LabVIEW configuration is the difference in termination characters. When received on either end of the communication port, the termination characters tell the port to stop reading. The LabVIEW serial configuration VI is capable of reading only one termination character, while the LS-100 requires two: a carriage return and a line feed character $(\mathrm{CR}+\mathrm{LF}$, in hexadecimal $0 \mathrm{D}$ is $\mathrm{CR}$, and $0 \mathrm{~A}$ is $\mathrm{LF})$. To ensure that the LS-100 communications are being received properly, each command sent to the LS-100 detector is appended with both characters. However, because only one character is read as the termination character by LabVIEW, in the configuration of the port using the "VISA Configure Serial Port" VI, only the final termination character is used-the Line Feed constant-as seen in Figure 1. This allows LabVIEW to read the entire serial string up to the final terminating character.

Additionally, within the initialization stage of the VI, the array and waveform used in plotting the continuous measurement data is initialized to zero. 



\section{2 Operational Stage}

The second step in the overall VI hierarchy is the operational stage, which is the " 1 " step in the overall sequence structure of the VI. Within this sequence, the program enters a "while" loop and continues operating within this operational stage until the user presses the "Stop/Abort Program" button on the front panel. Within this loop are three different operational sequences: the Detection Sequence, Mode Update Sequence, and Settings Configuration Sequence. Each is detailed below.

\section{2. 1 Detection Sequence}

Once the user presses either the "Single Measurement" or "Continuous Measurement," the detection sequence commences. Figure 2 shows the Detection sequence within the LabVIEW virtual instrument. Like almost all of the sequential operations within the VI there are three basic steps in the detection process: Send command to LS-100/110, Wait for detector to operate, and Listen for luminance reading/response. In the detection sequence there are also two additional steps, saving the data to file, and taking continuous measurements after the three basic steps. The detection sequence first sends the "MES" command (step 0), which tells the detector to measure luminance based on the mode it is operating in (See Mode Update Sequence for selecting modes).

The sequence then waits either 2.5 or 3 seconds depending on the selection of Slow or Fast detection in the mode selection (step 1). The LS-100/110 Luminance Meter Instruction manual [3] explains that it takes 0.8-1.0 t measure in "Fast" mode or 1.4-1.6 seconds to measure in "Slow" Mode, respectively. I found that the LabVIEW VI must wait $2.5 \mathrm{~s}$ in "Fast" mode and $3 \mathrm{~s}$ in Slow mode to correctly read the luminance data string from the LS-100/110 detector.

After the delay, the detection sequence reads the output string from the detector (step 2). The string is in the following format: [Error Code], [settings][Reading]. The luminance reading is pulled from the serial string and, depending on the unit character within the settings portion of the string, is output to the front panel with the correct units. Additionally, depending on the selection of the "Save to File?" button on the front panel, the data are then saved to file (step 3). Within this save-to-file step, the program first checks to see whether it has saved to file before. If not, it sets the current time to the reference time, and writes column headings to the top of the file. It then creates and saves a character string of the elapsed time between the reference time and measurement time, the luminance value, and the additional comment string. This last step is then repeated for each subsequent file save. When the user presses the "Reset Elapsed Reference Time in File to Current Time," the first step is repeated and a new reference time and column headings are appended to the data already stored to file.

Finally, if the user presses the "Continuous Measurement" button, the luminance value is plotted on the graph on the front panel and the program waits the user-defined number of seconds before exiting the detection sequence structure. With "Continuous Measurement" still pressed, the detection sequence is entered again; otherwise the program continues to loop within the operational "while" loop until another button is pressed. 


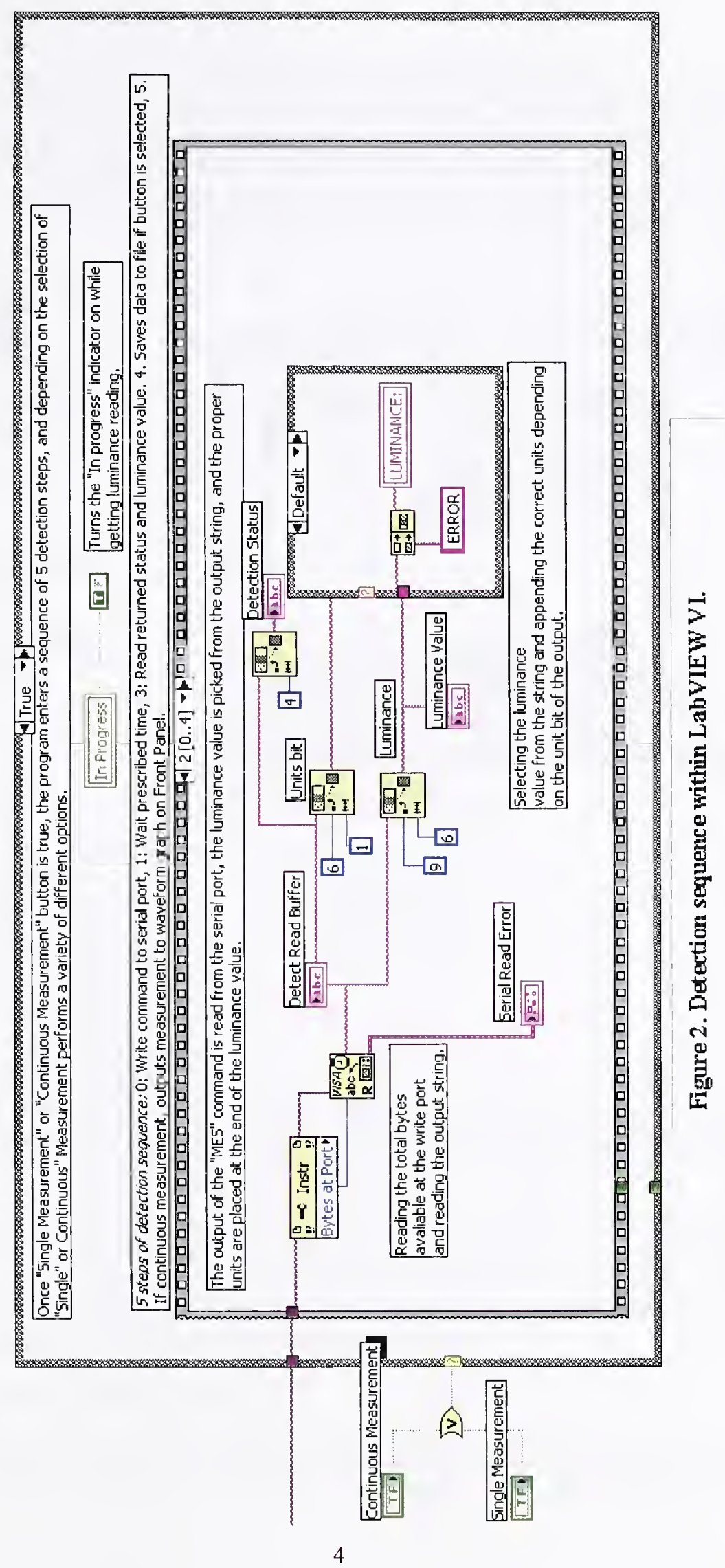





\section{2. 2 Mode Update Sequence}

The second sequence operating within the operational "while" loop is the "Mode Update" sequence. Once the user presses the "Update Modes" button, the sequence begins by first clearing the output status array on the front panel (step 0). This array will indicate whether the Sub-VI that was called in the next sequential step completed successfully or not (step 1). With the initialization completed, the program calls the Sub-VI "Luminance Detector Update Settings." This VI takes all of the settings within the "Mode Setup" Panel on the front panel, and updates the LS-100/110 to those settings. Figure 3 shows the "Mode Update" sequence.

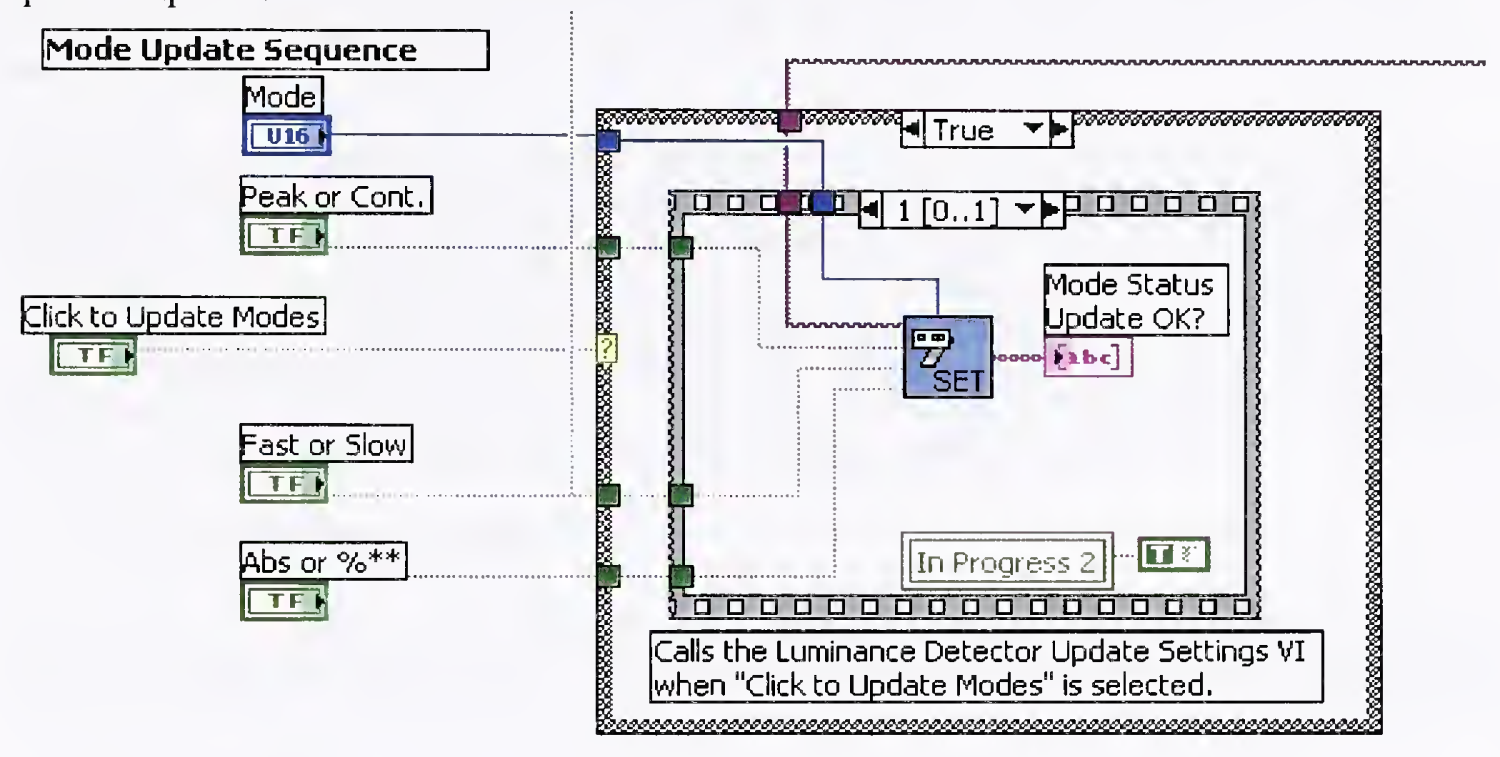

Figure 3. Mode update sequence.

There are four different calibration modes to select:

1. Preset calibration

2. User calibration

3. Color correction factor (C.C.F.)

4. Both user calibration and C.C.F.
(Preset Calibration)

(VARI-LUMI)

(VARI -C.C.F.)

(VARI -LUMI+C.C.F.);

as well as three additional "Mode" selections:

1. Fast or Slow detection

2. Peak or Continuous detection

3. Absolute or $\%$ readings.

The Sub VI completes a sequence of 16 steps consisting of selecting the appropriate command from the alternate choices depending on the "True" or "False" indication of the modes chosen followed by the normal send, wait, and receive process for sending all LS-100/110 commands.

With the completion of the 16 updating steps, the Sub-VI returns the "Mode Status Update Ok" array indicating whether all of the modes selected were updated successfully. These are indicated by the appropriate error codes. The most common error occurs when there is no $100 \%$ reference value or user calibration value stored in the detector and the \% or VARI modes are trying to be selected (See 3.2 Error Handling within 3. User Operation below for more information.)

\section{2. 3 Settings Configuration Sequence}

The last foundation of the LS-100/110 controller virtual instrument is the "Settings Configuration" sequence, which is shown in Figure 4. This sequence is controlled by the configuration buttons on the 

left sub-panel of the front panel. It allows the user to get and store $100 \%$ reference values, user calibration values, and color correction factors (C.C.F.).

The first step before the sequence starts is to create a string of eight digits, where each digit corresponds to the 1 or 0 value that represents the true or false value of the button pressed. This string creates a unique number $(1,10,100,1000,10000$ to 10000000 , where the leading zeros are ignored: $00000010=10)$ that represents a unique command sequence within the case structure on the right side of the figure. For each case, the program completes the desired commands of getting or storing values within the LS-100\110 detector memory using the same Send, Wait, and Receive pattern of the other sequences. The only deviations from this three-step sequence are added error-checking and user input before the command is sent in the four write commands.

The case statement is entered each time the user presses one of the configuration buttons. The functionality specific to that case is then performed and the program returns to iterating under the "while" loop until the next button is pressed. Error control has also been built into each of the cases that require numerical values to be entered. Before sending the command to the detector, the program checks the number of user-inputted digits to verify that the correct number of digits is being sent to the detector. If the number of digits is incorrect, the user is prompted to check the value and reenter the correct number of digits. Users are also asked to verify whether they want to overwrite the values. This prevents accidental overwriting if one of the configuration buttons is accidentally pressed.

\section{3 Close VISA Session Stage}

Once the user presses the "Stop/Abort Program" button, the VI exits from the "while" loop running within the operational stage and moves to the final step, where the program closes the VISA serial port and stops the operation of the VI. Figure 5 shows the LabVIEW diagram showing this "Close VISA" VI.

\section{User Operation}

Figure 6 below shows the control panel of the LabVIEW VI. It is composed of three separate sections: the main control box on the top, the "Advanced Configurations" box on the bottom, and the "Tabbed Info Box" to the right. The latter two can be hidden for a more compact display (see below for instructions). Through this control panel, the user performs the operation of the LS-100/110.

The first step in creating a connection between the detector and the LabVIEW controls is setting the detector to communication mode. The user does this by holding the "F" key down while turning on the LS-100\110. A "C" will appear in the lower right-hand corner of the display screen to indicate the detector is in communication mode. The user must also select the COM port within the LabVIEW VI where the detector is connected. In the "Tabbed Info Box" on the left side of the control panel under, the "RS-232 Comm. Error Indicators" tab, select the COM port where the detector is connected. It is advisable to right click and select "Make current value default" under "Data Operations" once the correct port has been selected. This will ensure that the port is correct each time the VI is opened and run. The current default is COM1.

With the connection established on the detector, the user can start the LabVIEW program. With the LabVIEW VI running, I recommend first verifying that the advanced configurations ( $100 \%$ reference value, User Calibration and C.C.R.) are stored as desired. Read the currently stored values by simply clicking the respective Get/Read buttons in the "Advanced Configurations" box. If they are not as desired, follow the procedures for storing the desired values below. With the settings as desired, the user can begin taking either single or continuous measurements and saving the data, all of which are also detailed under the operational procedures. 



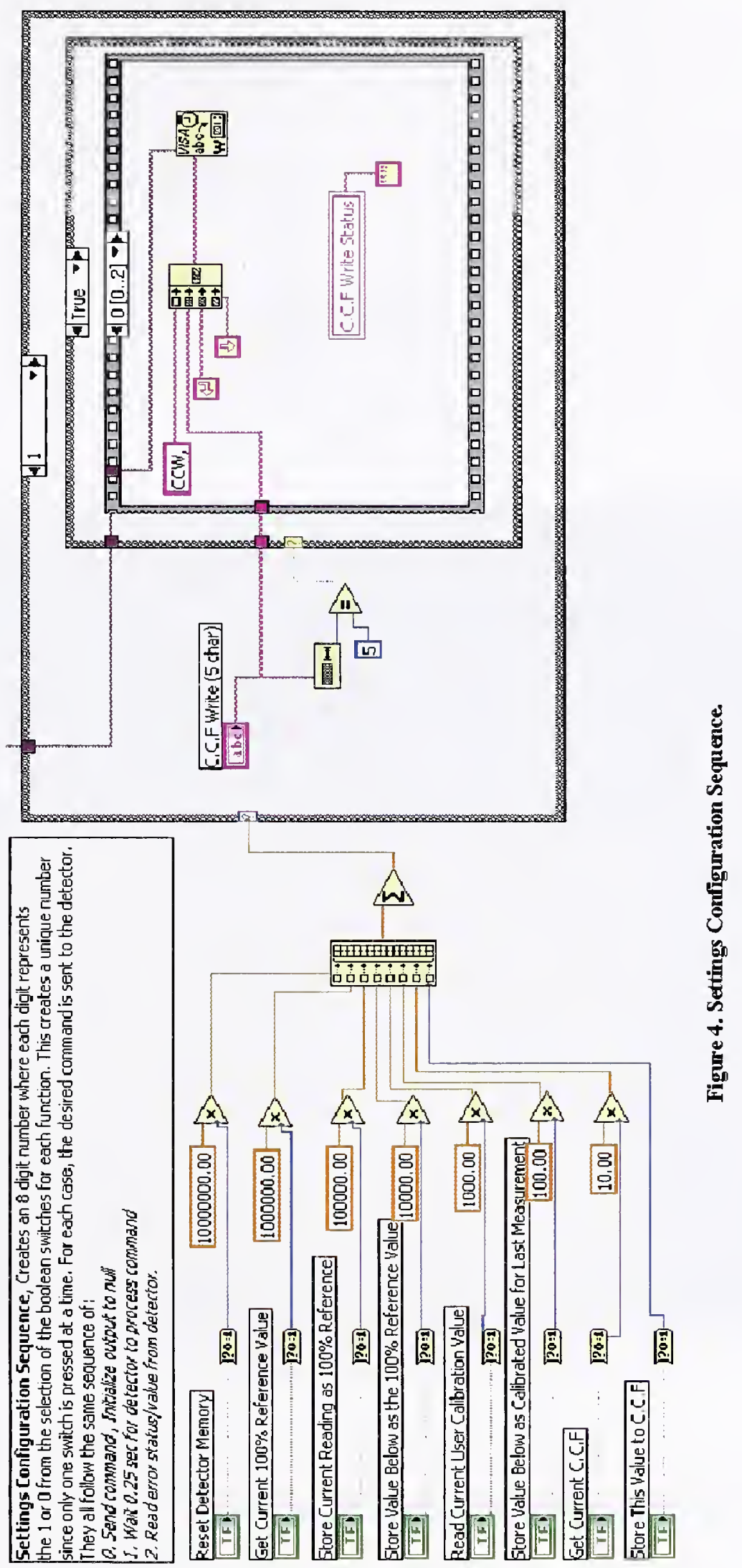





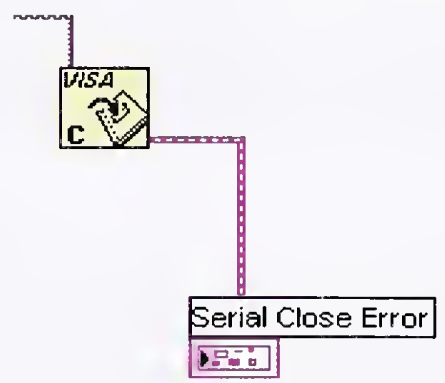

Figure 5. "Close Visa" VI.

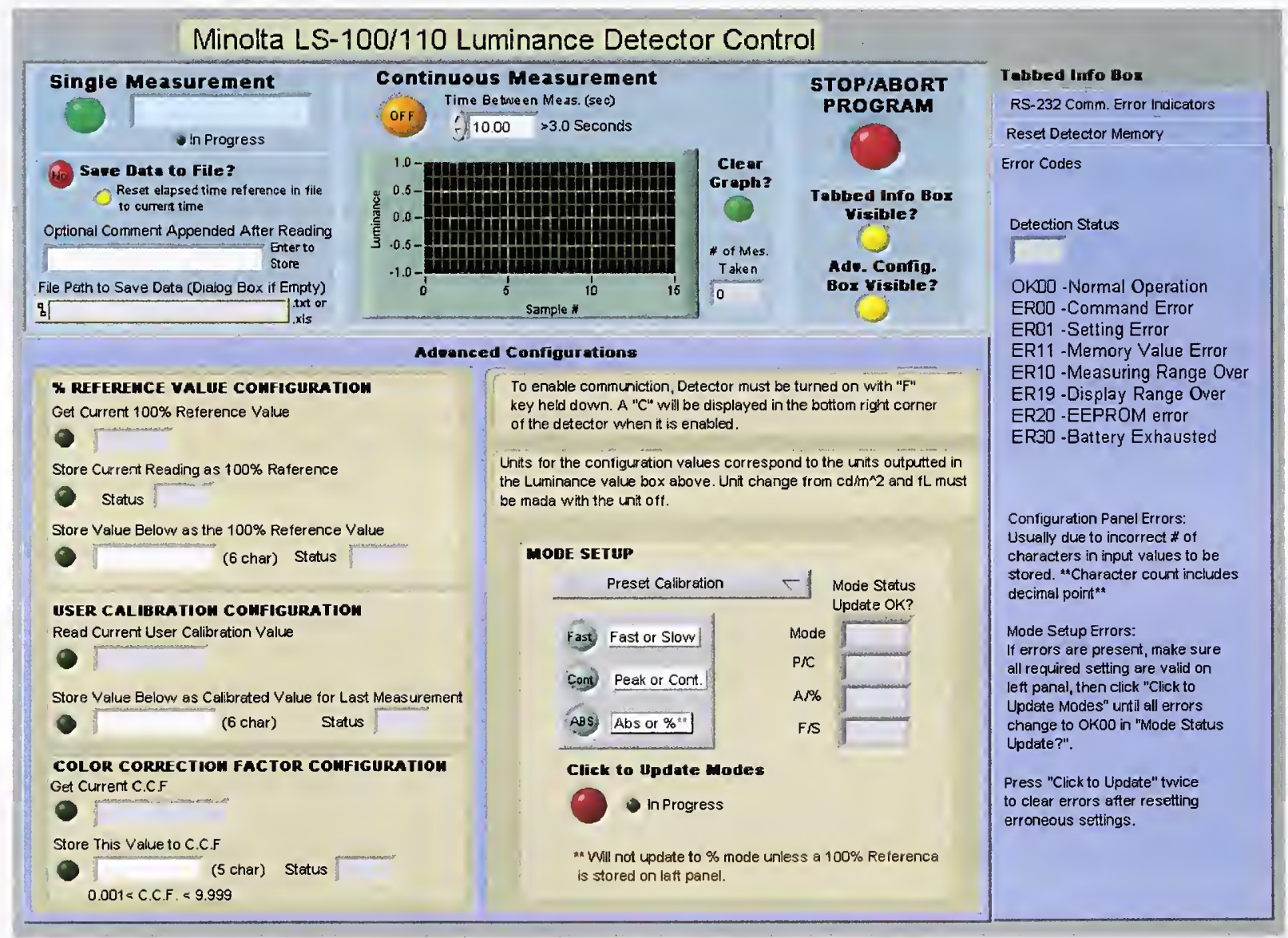

Figure 6. LS-100/110 LabVIEW control panel.

\section{1 Operational Procedures}

The steps for completing each of the LS-100/110 operations/functions are detailed within this section. 



\section{1. 1 Main Control Operations}

Single Measurement

1. Click on the "Single Measurement" Button. Measurement is being taken while "In Progress" light is on. Units of measurement are automatically added to the luminance reading based on the LS-100/110 setup.

Taking Continuous Measurements

1. Set the desired time between each measurement. This value must be greater than $3 \mathrm{~s}$, but can be changed in between measurements if desired.

2. Click on the "Continuous Measurement" button. Each measurement is plotted to show any trends. To clear plot, press the "Clear Graph?" button. This resets the plot and the "\# of Meas. taken" count to zero.

Saving Data to File

1. Press "Save Data to File?" button (top left, below "Single Measurement" button)

2. Enter the file path where the data is to be saved (.txt or .xls). If no path is entered, a dialog box will pop up and the user will be asked to select a file. Either the file can currently exist or LabVIEW will create it. If the file already exists, the data will be appended to the end of the contents already present in the file.

3. Insert a comment, if desired, to distinguish measurements within the file. To insert, type in the desired text and press "Enter" or click outside of the text box.

4. Begin taking measurements; the data will be saved to file with the elapsed time since data began being taken, the luminance value with units, and the additional user-added comment, if desired, all saved on a single line in the file.

5. Clicking "Reset elapsed time reference value in file to current time" button will reset the time from which the elapsed time value in the file is calculated for each measurement. This button is visible only after the first luminance measurement is saved to file.

Note: Data can be saved only if the "Save Data to File?" button is illuminated before the measurement is taken.

Hiding "Advanced Configuration" and "Tabbed Info" boxes

1. Press "Tabbed Info Box Visible?" or "Adv. Config. Box Visible?" button Boxes will disappear until buttons are pressed again.

Note: A delay in the hiding/reappearance may occur because the program must first finish whatever sequence it is in before checking the hide/visible settings within the operational while loop.

Stopping/Aborting Program.

1. Press "Stop/Abort Program" button

2. Program will finish whatever programming sequence it is within and then stop; there might be a delay while it finishes the sequence.

\section{1. 2 Advanced Configuration Operations}

Storing Current Reading as $100 \%$ reference.

1. Take single measurement by pressing the "Single Measurement" button

2. Click "Store Current Measurement as $100 \%$ reference" button

3. Change mode from ABS to $\%$ and update modes (see Updating Modes)

4. Begin taking $\%$ measurements 

Storing Nominal value as $100 \%$ reference

1. Enter desired value

2. Click "Store Value Below as the $100 \%$ Reference Value" button

3. Change mode from ABS to \% and update modes (see Updating Modes)

4. Begin taking \% measurements.

Storing User Calibration Value

1. Take single measurement by pressing the "Single Measurement" button

2. Enter desired calibration value

3. Click "Store Value Below as Calibration for Last Measurement" button

4. To use calibration values, select VARI-LUMI or VARI-C.C.F+LUMI mode on the pull down menu in the "Mode Setup" panel and update modes (see Updating Modes)

Storing C.C.F value

1. Enter value

2. Click "Store This Value to C.C.F." button

3. To use C.C.F select VARI-C.C.F or VARI-C.C.F +LUMI mode on the pull down menu in the

"Mode Setup" panel and update modes (see Updating Modes)

Updating Modes

1. Select the desired settings on the "Mode Setup" panel and click "Click to Update Modes"

button. When indicator goes out, update is complete.

2. If "Mode Status Update Ok?" indicator shows an error, verify that all required configuration values are stored in the configuration panels.

3. Click "Click to Update Modes" button until all rows show only "OK00".

\section{1. 3 Tabbed Info Box Operations}

\section{Clearing Memory}

1. Select "Reset Detector Memory" tab on "Tabbed Info Box" on right side

2. On this tab, click "Reset Detector Memory" button

3. Verify memory is to be cleared in pop-up box. When memory reset is complete, an "OK00" status will be displayed next to "Reset Detector Memory" button

4. If using \% or LUMI modes, re-store necessary configuration values or change from $\%$ and LUMI modes, which depend on erased configuration data (100\% reference and User Calibration values) now set to 0.00 . Not doing so will cause detection errors.

\section{2 Error handling}

The first step to ensuring error-free detection is to always set the modes and configurations before taking measurements. By ensuring that all modes are displaying the "OK00" normal operation indication and that the required configuration values are all stored, detection will be trouble free. If "Error" does appear in the "Luminance" display, follow these steps:

1. Make sure the "C" is present in the LS-100/110 lower right corner. See User Operation section if not.

2. Get/Read all of the configuration values on the left panel of the "Advanced Configuration" box.

3. If there is a zero value present on one of the configuration values, verify that the current mode (Preset, C.C.F., or LUMI) being used does not depend on the value being nonzero (100\% Reference and User Calibration values).

4. Change mode to a setting where this configuration will not be a problem 

5. Click "Click to Update Modes" until error is clear and "Ok00" shows on all four rows of the "Mode Status Update OK?" Indicator.

(Clicking "Click to Update Modes" twice is usually required, once to clear the error display on the detector, and once more to reset the mode configuration.)

6. Re-Set configurations for desired operations using operational procedures above.

7. Take a measurement and verify detector side panel value and Luminance value on Virtual Instrument control panel match. If not, press "Update Modes until all display "OK00", and repeat until VI is reading in real time. Clicking "Update Modes" clears the erroneous data from the write buffer of the device.

In addition, several error indicators are also built into the front panel of the detector. Under the "RS-232 Comm. Error Indicators" tab in the "Tabbed Info Box" the right side of the diagram are error indication boxes for each of the four serial communication steps: open, detection read and write, and closing of the port. By right clicking on the error codes in the boxes, it is possible to get information about what is causing the communication error. Additionally, error outputs are available for each serial read and write process within the diagram, but were not included on the front panel because of their large number. They can be probed if troubleshooting is required by selecting the probe tool in the wiring diagram tools pallet and clicking the error output of any of the VISA Read/Write VIs in the diagram.

Also under the same "RS-232 Comm. Error Indicators" are the VISA Port selection box, (default set to COM1), and the full output from the read buffer during detection, which is useful to verify the output is as it should be according to the LS-100/110 Communication Manual.

\section{Summary}

Through the use of LabVIEW Virtual Instruments, it is possible to create a fully functional controller for a Minolta LS-100 luminance detector. The LabVIEW VI can take single or continuous luminance measurements using any of the built-in features of the LS-100/110. Additionally, the data can be saved to a text or spreadsheet file, which allows for easier data acquisition and manipulation over reading and writing the values from the detector display.

\section{References}

[1] Certain commercial equipment, software, instruments, materials, systems, and trade names are identified in this paper only in order to specify or identify technologies adequately. Such identification implies neither recommendation nor endorsement by the National Institute of Standards and Technology, nor is it intended to imply that the systems or products identified are necessarily the best available for the purpose.

[2] Minolta Co, Ltd. Luminance Meter LS-100/110 Communication Manual. Japan: Minolta Co, Ltd, 2000.

[3] Minolta Co, Ltd. Luminance Meter LS-100/110 Instruction Manual. Japan: Minolta Co, Ltd, 1987. 
